Research Article

\title{
Periodic Attitudes of Libration Point Spacecrafts in the Earth-Moon System
}

\author{
Qiwei Guo, ${ }^{1}$ Bo Xu $\mathbb{D}^{2},{ }^{2}$ and Hanlun Lei $\mathbb{D}^{1}$ \\ ${ }^{1}$ School of Astronomy and Space Science, Nanjing University, Nanjing 210046, China \\ ${ }^{2}$ School of Aeronautics and Astronautics, Sun Yat-Sen University, Guangzhou 510275, China \\ Correspondence should be addressed to Bo Xu; xubo27@mail.sysu.edu.cn
}

Received 25 July 2019; Revised 11 March 2020; Accepted 16 March 2020; Published 29 April 2020

Academic Editor: Fazal M. Mahomed

Copyright (c) 2020 Qiwei Guo et al. This is an open access article distributed under the Creative Commons Attribution License, which permits unrestricted use, distribution, and reproduction in any medium, provided the original work is properly cited.

The attitude motion of a rigid spacecraft is studied in the Earth-Moon circular restricted three-body problem. Firstly, the equilibrium attitude and its stability as functions of the moments of inertia are discussed when the spacecraft is assumed at the libration points. Then, periodic attitudes of a spacecraft with mass distribution given in the stable regions are obtained. Regarding space mission applications, the Sun orientation is discussed, and the orbit-attitude resonances are constructed for spacecrafts working on the libration point orbits by means of a continuation procedure.

\section{Introduction}

In recent decades, the dynamics about the libration points in the circular restricted three-body problem (CRTBP) has been studied extensively [1-17], and so far, several libration point missions have been conducted [18-24]. As we know, both orbital and attitude motions of a spacecraft are required to be considered in practical missions. However, the attitude motion near the libration point has attracted few attentions. In the environment of CRTBP, understanding the natural attitude motion may offer options that lessen the control effort for the attitude control system. In the proper circumstances, the natural dynamical revolution may assist, or even replace, the attitude control action [25]. Thus, to design the spacecraft control system better, an understanding of the coupling orbital and attitude motion is of significance.

Kane and Marsh [26] considered the attitude stability of an axial symmetric satellite which is located at the libration points. The satellite is spinning about its axis of symmetry that is normal to the primary bodies' orbital plane. Later, Robinson [27] studied the attitude stability of a dumbbell satellite located at the equilateral points. The same author [28] also considered the attitude stability of an arbitraryshaped rigid satellite which is located at a Lagrangian point, and the author constructed a linear stability diagram about the stable attitude of the satellite. Abad et al. [29] introduced the use of Euler parameters in the study of a rigid body located at L4. Brucker and Gurfil [30] identified the dynamics and stability of a rigid body spacecraft at the collinear Lagrange points using Poincare maps.

It is more probable that a spacecraft is not exactly placed at a Lagrangian point but located on a periodic orbit. Recently, several authors have investigated the attitude motion of a spacecraft on planar or three-dimensional periodic orbits. Using the Euler angle pitch, roll, and yaw, Wong et al. [31] introduced how to construct the rotational dynamical equations of a rigid body. The stability corresponding to different mass distributions is shown at Sun-Earth Lagrangian L2 and L4, and the effects of orbital motion on its attitude dynamics in the vicinity of a collinear Lagrangian point are investigated. Knutson et al. [32] took much effort to a better understanding of the attitude dynamics for a rigid spacecraft within the CRTBP, and their analysis is expanded to fully nonlinear orbits while limiting the orbital motion to the planar Lyapunov families about Earth-Moon libration points L1 and L2. In the Earth-Moon system, Guzzetti and Howell [33] investigated a general approach to compute orbit-attitude periodic solutions that are associated with known CRTBP reference orbits (L1/L2 Lyapunov orbits, DROs). Meng et al. [34] analysed the attitude motion of a 
dual-spin satellite in various halo orbits, and a semianalytical expansion of the gravity torque is employed to explain the mechanism of attitude motion. Bucci et al. [35] studied the planar orbit-attitude dynamics in both a standard and a perturbed CRTBP environment. Colagrossi and Lavagna [36] investigated the coupling between orbit-attitude dynamics and the flexibility of the structure in a slightly different way than the one presented in this paper. More recently, to construct a basis for understanding attitude motion within a multibody problem with application to spacecraft flight dynamics, a review about attitude dynamics in the CRTBP was made by Guzzetti and Howell [25].

Recently, using numerical techniques, Lei et al. [37] studied the periodic attitudes and bifurcation for a rigid spacecraft on a stationary orbit around a uniformly rotating asteroid. Using the same method, in this present work, we aim to construct the attitude motions of a rigid spacecraft moving at and around the libration points in the CRTBP. Euler angles in terms of the 3-2-1 rotation sequence that describes the relationship between the body-fixed frame with respect to the orbital frame are taken to define the attitude of a rigid spacecraft. The equilibrium attitudes and the periodic attitude motion around them are obtained for rigid spacecrafts working at the Earth-Moon libration points. Based on these solutions, periodic attitude motions along the libration point orbit are discussed. Periodic motions of the orbit and attitude can be a novel contribution to the exploration of the CRTBP, and it could serve as a stepping stone for more dynamically complex environment. Considering that the solar-sail spacecraft has been studied extensively [38-41], the Sun orientation is also investigated for the special type of spacecraft effectively using radiation pressure on a reflective sail for propulsion.

This paper is organized as follows. In Section 2, the dynamical model of the CRTBP is introduced first, and the rotational equations are given. In Section 3, the equilibrium attitude and its stability corresponding to different shaped rigid bodies are constructed when the spacecraft is located at a given libration point. And, the periodic attitude motion around the stable equilibrium attitude is obtained. Then, the Sun orientation and the orbit-attitude resonances of a spacecraft along a Lyapunov orbit around libration point L4 are shown in Section 4. Section 5 concludes this work.

\section{Dynamical Models}

In this section, we briefly introduce the basic dynamical models used in this work.

\subsection{The Circular Restricted Three-Body Problem. In this} paper, we consider a spacecraft moving in the CRTBP, which describes the motion of a spacecraft $P_{3}$ with infinitesimal mass under the gravitational attraction of two massive bodies $P_{1}$ with mass $m_{1}$ and $P_{2}$ with mass $m_{2}$, moving in circular orbits around their barycentre. Without loss of generality, we assume that $m_{1}>m_{2}$, and in this case, we call $P_{1}$ as the massive primary and $P_{2}$ as the secondary. Usually, the motion of $P_{3}$ is described in a barycentric rotating coordinate system (see Figure 1), in which the origin coincides with the mass centre of the primaries, the $x$-axis is directed from $P_{1}$ towards $P_{2}$, the $z$-axis is parallel to the angular momentum vector of the primaries, and the $y$-axis is determined by the right-handed coordinate frame.

For consideration of computation accuracy, the variables are normalized by taking the distance between the primaries, their total mass, and orbital period divided by $2 \pi$ as the units of length, mass, and time, respectively. Under this normalized system, the mass of the secondary is $\mu=\left(m_{2} /\left(m_{1}+m_{2}\right)\right)$, and the mass of the primary is $1-\mu$. In the barycentric coordinate frame, the primary and secondary bodies are located at $(-\mu, 0)$ and $(1-\mu, 0)$, respectively.

In this paper, we only consider the planar orbital motion of the spacecraft. The differential equations, governing the motion of the spacecraft, can be written as [42]

$$
\begin{aligned}
& \ddot{x}-2 \dot{y}=\frac{\partial \Omega}{\partial x}, \\
& \ddot{y}+2 \dot{x}=\frac{\partial \Omega}{\partial y},
\end{aligned}
$$

where

$$
\Omega=\frac{1}{2}\left(x^{2}+y^{2}\right)+\frac{1-\mu}{r_{1}}+\frac{\mu}{r_{2}},
$$

is the effective potential of the CRTBP. The distance of the spacecraft from the primaries is $r_{1}$ and $r_{2}$ given by

$$
\begin{array}{r}
r_{1}=\sqrt{(x+\mu)^{2}+y^{2}}, \\
r_{2}=\sqrt{(x-1+\mu)^{2}+y^{2}} .
\end{array}
$$

It is known that (1) possesses a conserved quantity, known as Jacobi integral,

$$
C=2 \Omega-\left(\dot{x}^{2}+\dot{y}^{2}\right)
$$

and five Lagrangian libration points exist in the model specified by (1). Three of them lying on the $x$-axis are denoted as collinear libration points L1, L2, and L3. The remaining points denoted by L4 and L5 form equilateral triangles with the primary bodies.

2.2. Rotational Model. Now, we reconsider that the spacecraft is not a point mass but a finite-size rigid body moving nearby a libration point in the CRTBP. It means that the infinitesimal point mass is replaced by a finite-size one. The orbital motion of the spacecraft can be described in the reference frame $o-x y z$ (refer to Figure 1). The attitude motion is described by the orientation difference between the orbital reference frame and the body-fixed reference frame $p-x_{B} y_{B} z_{B}$ (see Figure 1) which is aligned with the principal axes of the body. The relationship between the body-fixed frame with respect to the orbital frame is measured in the 3-2-1 (pitch-roll-yaw) rotation sequence with the Euler angles $(\theta, \phi$, and $\psi)$. Since that the dimensions of the spacecraft are too small compared with its distance to each of the primaries, the attitude has negligible influence 


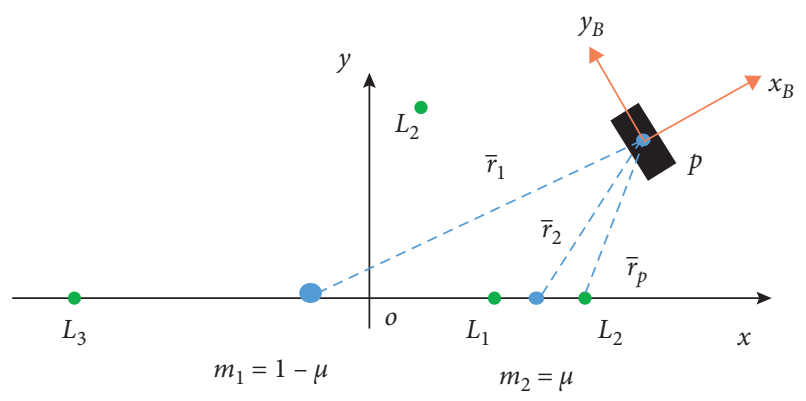

$\stackrel{\bullet}{L}_{5}$

FIGURE 1: The barycentric rotating reference frame $o-x y$ and body-fixed frame $p-x_{B} y_{B}$.

upon the orbital motion so that we can assume that the orbital motions are decoupled with the attitude motions.

For the spacecraft moving nearby a libration point, the gravitational gradient torques exerted by the primary bodies are the dominant torques acting on a spacecraft. The moments of inertia of the spacecraft about the body principal axes $x_{B}, y_{B}$, and $z_{B}$ are denoted by $I_{x x}, I_{y y}$, and $I_{z z}$, respectively. The first-order gravity gradient torque exerted by the $i$-th primary body about the spacecraft's centre of mass can be written as [31]

$$
\left\{\begin{array}{l}
M_{x B}=\frac{3 \mu r_{i z B} r_{i y B}}{r_{i}^{3}}\left(I_{z z}-I_{y y}\right) \\
M_{y B}=\frac{3 \mu r_{i x B} r_{i z B}}{r_{i}^{3}}\left(I_{x x}-I_{z z}\right) \\
M_{z B}=\frac{3 \mu r_{i y B} r_{i x B}}{r_{i}^{3}}\left(I_{y y}-I_{x x}\right) .
\end{array}\right.
$$

In (5), $r_{i x B}, r_{i x B}$, and $r_{i x B}(i=1$ or $i=2)$ are the components of the unit vector along $r_{i B}$ which is the distance from the $i$-th primary body to the centre mass of the spacecraft expressed in body-fixed frame $p-x_{B} y_{B} z_{B}$.

Considering the angular velocity $\left(\omega_{x}, \omega_{y}, \omega_{z}\right)$ of a spacecraft in the inertial frame, there are six state variables $\left(\omega_{x}, \omega_{y}, \omega_{z}, \psi, \phi\right.$, and $\left.\theta\right)$ to describe its attitude motion in this paper. With the same nondimensional unit as the orbital motion, the kinematic differential equations are described as [31]

$$
\left\{\begin{array}{l}
\dot{\omega}_{x}=-k_{1}\left(\omega_{y} \omega_{z}-\frac{3(1-\mu)}{r_{1}^{3}} r_{1 z B} r_{1 y B}-\frac{3 \mu}{r_{2}^{3}} r_{2 z B} r_{2 y B}\right), \\
\dot{\omega}_{y}=k_{2}\left(\omega_{z} \omega_{x}-\frac{3(1-\mu)}{r_{1}^{3}} r_{1 x B} r_{1 z B}-\frac{3 \mu}{r_{2}^{3}} r_{2 x B} r_{2 z B}\right), \\
\dot{\omega}_{z}=-k_{3}\left(\omega_{x} \omega_{y}-\frac{3(1-\mu)}{r_{1}^{3}} r_{1 y B} r_{1 x B}-\frac{3 \mu}{r_{2}^{3}} r_{2 y B} r_{2 x B}\right)
\end{array}\right.
$$

where

$$
\begin{aligned}
& k_{1}=\frac{I_{z z}-I_{y y}}{I_{x x}}, \\
& k_{2}=\frac{I_{z z}-I_{x x}}{I_{y y}}, \\
& k_{3}=\frac{I_{y y}-I_{x x}}{I_{z z}},
\end{aligned}
$$

are the inertia ratios. Euler's equations are solved together to obtain the complete rotational dynamics of the rigid spacecraft [31]:

$$
\left[\begin{array}{c}
\dot{\psi} \\
\dot{\phi} \\
\dot{\theta}
\end{array}\right]=\frac{1}{\cos \phi}\left[\begin{array}{ccc}
\cos \phi & \sin \phi \sin \psi & \sin \phi \cos \psi \\
0 & \cos \phi \cos \psi & -\cos \phi \sin \psi \\
0 & \sin \psi & \cos \psi
\end{array}\right]\left[\begin{array}{c}
\omega_{B}^{x} \\
\omega_{B}^{y} \\
\omega_{B}^{z}
\end{array}\right],
$$

where $\left(\omega_{B}^{x}, \omega_{B}^{y}, \omega_{B}^{z}\right)$ are the angular velocities of the spacecraft measured in the Earth-Moon rotating frame. The relationship of $(\psi, \phi, \theta),\left(\omega_{x}, \omega_{y}, \omega_{z}\right)$, and $\left(\omega_{B}^{x}, \omega_{B}^{y}, \omega_{B}^{z}\right)$ can be transformed by $[31,37]$

$$
\left[\begin{array}{c}
\omega_{B}^{x} \\
\omega_{B}^{y} \\
\omega_{B}^{z}
\end{array}\right]=\left[\begin{array}{c}
\omega_{x}+\sin \phi \\
\omega_{y}-\cos \phi \sin \psi \\
\omega_{z}-\cos \phi \cos \psi
\end{array}\right]=\left[\begin{array}{c}
\dot{\psi}-\dot{\theta} \sin \phi \\
\dot{\theta} \cos \phi \sin \psi+\dot{\phi} \cos \psi \\
\dot{\theta} \cos \phi \cos \psi-\dot{\phi} \sin \psi
\end{array}\right] .
$$

\section{Attitude Motion at the Earth-Moon Libration Points}

Considering the Earth-Moon system as a case study in the part, the attitude motions of spacecrafts at L2 and L4 are studied.

\subsection{Libration Point L2}

3.1.1. Equilibrium Attitude and Its Stability. Considering that a spacecraft is located at libration point $\mathrm{L} 2$, it is known that the equilibrium attitude has zero Euler angles, i.e., $\left(\psi_{E}, \phi_{E}, \theta_{E}\right)=(0,0,0)[25,31]$. By denoting the state relative to the equilibrium attitude as $(\Delta \psi, \Delta \phi, \Delta \theta)$, the Euler angles of the spacecraft can be expressed by $\psi=\psi_{E}+\Delta \psi$, $\phi=\phi_{E}+\Delta \phi$, and $\theta=\theta_{E}+\Delta \theta$. Around the equilibrium attitude, (6) can be linearized as follows [31];

$$
\left\{\begin{array}{l}
\Delta \ddot{\psi}+\left(k_{1}-1\right) \Delta \dot{\phi}+k_{1} \Delta \psi=0, \\
\Delta \ddot{\phi}+\left(1-k_{2}\right) \Delta \dot{\psi}+(3 B+1) k_{2} \Delta \phi=0, \\
\Delta \ddot{\theta}+3 B k_{3} \Delta \theta=0,
\end{array}\right.
$$

where

$$
\begin{gathered}
B=\frac{1-\mu}{R_{1 L}^{3}}+\frac{\mu}{R_{2 L}^{3}}, \\
k_{3}=\frac{k_{2}-k_{1}}{1-k_{1} k_{2}} .
\end{gathered}
$$


By analysing the characteristic equations of linear equation (10), the stability conditions can be written as $[25,28]$

$$
\left\{\begin{array}{l}
C^{2}-D>0 \\
C>0 \\
D>0 \\
k_{3}>0
\end{array}\right.
$$

where $C=3 B k_{2}+k_{1} k_{2}+1$, and $D=4(3 B+1) k_{1} k_{2}$.

Then, the stability diagram on the $k_{2}-k_{1}$ plane for spacecrafts located at L2 is shown in Figure 2(a), and coloured regions I and II represent that the equilibrium attitude is stable with the corresponding mass distribution. The stable domains are distributed in the first and third quadrants, similar to the conclusions given by Guzzetti and Howell, Robinson, and Wong et al. [25, 28, 31]. The stability domain in the first quadrant is a triangular region which represents $k_{2}>k_{1}>0\left(I_{z z}>I_{y y}>I_{x x}\right)$, and in the third quadrant, the stability domain represents $0>k_{2}>k_{1}\left(I_{y y}>I_{x x}>I_{z z}\right)$.

3.1.2. Periodic Attitudes and Their Stability. In this part, we take a spacecraft with mass distribution in region $\mathrm{I}\left(k_{1}=\right.$ 0.2 and $\left.k_{2}=0.4\right)$ as an example. The linear solution of attitude motion around the equilibrium attitude $\left(\psi_{E}, \phi_{E}, \theta_{E}\right)$ can be written as $[31,37]$

$$
\left\{\begin{array}{l}
\Delta \psi=\alpha_{1} \cos \left(w_{1} t+\varphi_{10}\right)+\alpha_{2} \cos \left(w_{2} t+\varphi_{20}\right), \\
\Delta \phi=\bar{\kappa}_{1} \alpha_{1} \sin \left(w_{1} t+\varphi_{10}\right)+\bar{\kappa}_{2} \alpha_{2} \sin \left(w_{2} t+\varphi_{20}\right), \\
\Delta \theta=\alpha_{3} \cos \left(w_{3} t+\varphi_{30}\right),
\end{array}\right.
$$

where $\alpha_{1}$ and $\alpha_{2}$ are amplitudes in the yaw and roll directions, $\alpha_{3}$ is the amplitude of the pitch motion, and $\varphi_{10}, \varphi_{20}$, and $\varphi_{30}$ are the corresponding initial phase angles. In (13), $\omega_{1}, \omega_{2}$, and $\omega_{3}$ are the frequencies of attitude motion, which are determined by

$$
\left\{\begin{array}{l}
\omega_{1}=\frac{\sqrt{2}}{2} \sqrt{C-\sqrt{C^{2}-D}}, \\
\omega_{2}=\frac{\sqrt{2}}{2} \sqrt{C+\sqrt{C^{2}-D}}, \\
\omega_{3}=\sqrt{3 B k_{3}},
\end{array}\right.
$$

and the constant coefficients are determined by

$$
\begin{gathered}
\bar{\kappa}_{1}=-\frac{k_{1}-\omega_{1}^{2}}{\left(k_{1}-1\right) \omega_{1}}, \\
\bar{\kappa}_{2}=-\frac{k_{1}-\omega_{2}^{2}}{\left(k_{1}-1\right) \omega_{2}} .
\end{gathered}
$$

In this case, there are three types of basic periodic attitudes emanating from the equilibrium attitude. Figure 3 represents the configurations of these types of periodic attitudes with $\alpha_{1}=0.1,0.2,0.3 \mathrm{deg}, \alpha_{2}=0.1,0.2,0.3 \mathrm{deg}$, and $\alpha_{3}=0.1,0.2,0.3 \mathrm{deg}$, respectively.
Based on these linear solutions, we construct the corresponding periodic attitudes (see Figure 4) in the CRTBP model by means of numerical techniques. The state of attitude is denoted by $(\psi, \phi, \theta, \dot{\psi}, \dot{\phi}, \dot{\theta})$, and the corresponding period is T. If only an initial state of attitude is known, we can obtain the periodic attitude motion by integrating (6) over one period.

To generate a family of periodic solution, the well-known continuation scheme called pseudo-arclength continuation $[37,43]$ is used in this investigation. Based on these linear solutions in Figure 4, the corresponding attitude families are obtained shown in the Euler angle $(\psi, \phi, \theta)$ space (see the first family in Figure 5(c)). As it is shown in Figure 6, three families of periodic attitudes have been constructed. The attitude periods of the families are extended in the range of [64.584, 70.389] days, [12.557, 13.301] days, and [18.928, 20.199] days.

Meanwhile, we obtained the stability of these periodic attitude motions. The stability of the periodic attitude can be reflected by its monodromy matrix, which corresponds to the state transition matrix over one period [37, 44]. Three reciprocal pairs of eigenvalues [45] exist in the periodic attitude motion which is an autonomous and Hamiltonian system. Among the three pairs of eigenvalues, there are two pairs of eigenvalues which are denoted by $\left(s_{1}, s_{2}\right)$ and $\left(s_{3}, s_{4}\right)$ with $s_{1}=\left(1 / s_{2}\right)$ and $s_{3}=\left(1 / s_{4}\right)$, and the other pair of eigenvalues is unitary, i.e., $s_{5}=s_{6}=1$. Similar to the stability index defined for the periodic orbits in the CRTBP [46-48], two stability indices in attitude motion are defined as $s_{h}=s_{1}+s_{2}$ and $s_{v}=s_{3}+s_{4}$. If both conditions $s_{h}<2$ and $s_{v}<2$ are satisfied, the periodic attitude is stable; otherwise, it is unstable. The tangent bifurcations occur when $s_{h}=2$ or $s_{v}=2$, and the period-doubling bifurcations take place when $s_{h}=-2$ or $s_{v}=-2$. For the first attitude family, two pairs of eigenvalues $\left(s_{1}, s_{2}\right)$ and $\left(s_{3}, s_{4}\right)$ are shown in Figure 5(a), and two stability indices $s_{h}$ and $s_{v}$ are shown in Figure 5(b). From Figure 5, we can conclude that the periodic attitudes corresponding to the first attitude family are always stable. The Euler angles along the evolution of the first attitude family are shown in Figure 5(c).

\subsection{Libration Point L4}

3.2.1. Equilibrium Attitude and Its Stability. Similarly, we obtain the equilibrium attitude of the rigid spacecraft fixed at libration point L4 with $\left(\psi_{E}, \phi_{E}, \theta_{E}\right)=(0,0,-(\arctan \sqrt{3}$ $(1-2 \mu) / 2))$. And the Euler angles of the spacecraft can be expressed by $\psi=\psi_{E}+\Delta \psi, \phi=\phi_{E}+\Delta \phi$, and $\theta=\theta_{E}+\Delta \theta$ by denoting the state relative to the equilibrium attitude as $(\Delta \psi, \Delta \phi, \Delta \theta)$. Then, (6) could be linearized about the equilibrium attitude at L4 as follows [31]:

$$
\left\{\begin{array}{l}
\Delta \ddot{\psi}+\left(k_{1}-1\right) \Delta \dot{\phi}+L k_{1} \Delta \psi=0, \\
\Delta \ddot{\phi}+\left(1-k_{2}\right) \Delta \dot{\psi}+M k_{2} \Delta \phi=0, \\
\Delta \ddot{\theta}+(2 M-5) k_{3} \Delta \theta=0,
\end{array}\right.
$$

where 


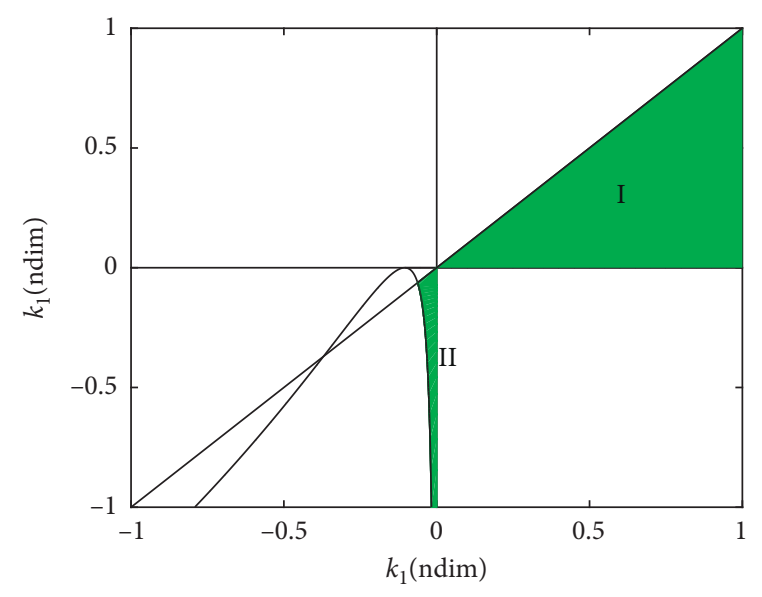

(a)

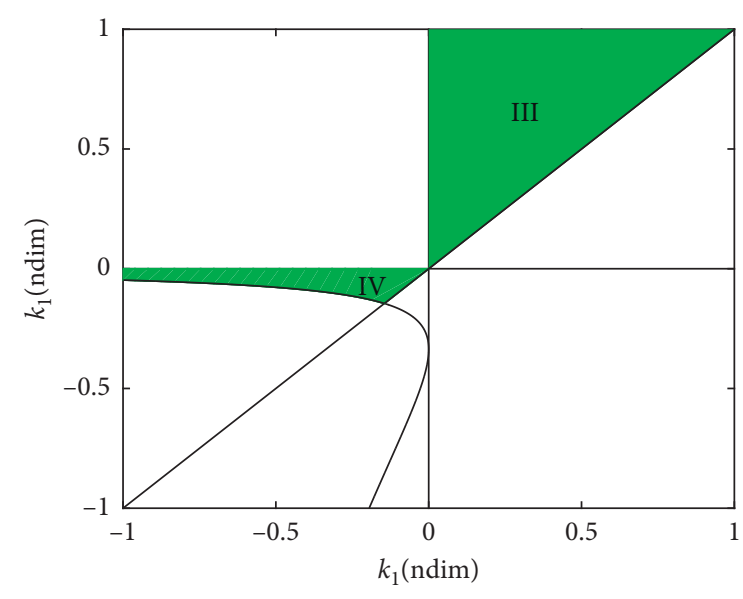

(b)

Figure 2: Stability diagram for a rigid spacecraft at libration points L2 (left panel) and L4 (right panel) in the Earth-Moon system.

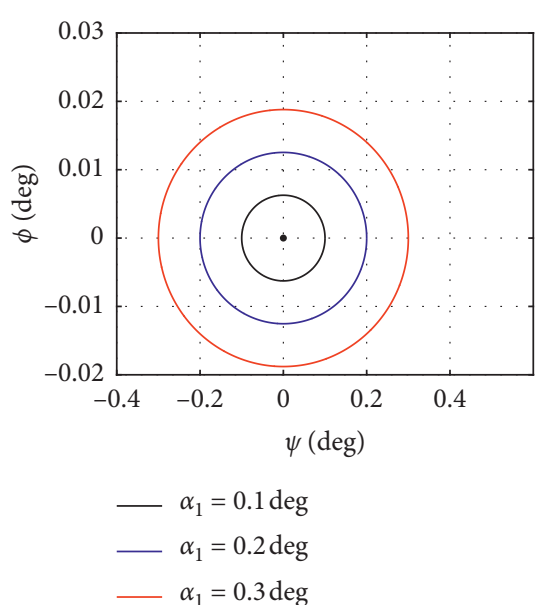

(a)

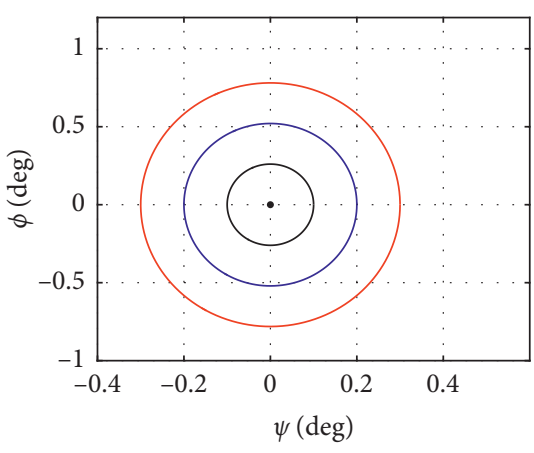

$\begin{aligned}-\alpha_{2} & =0.1 \mathrm{deg} \\ -\alpha_{2} & =0.2 \mathrm{deg} \\ -\alpha_{2} & =0.3 \mathrm{deg}\end{aligned}$

(b)

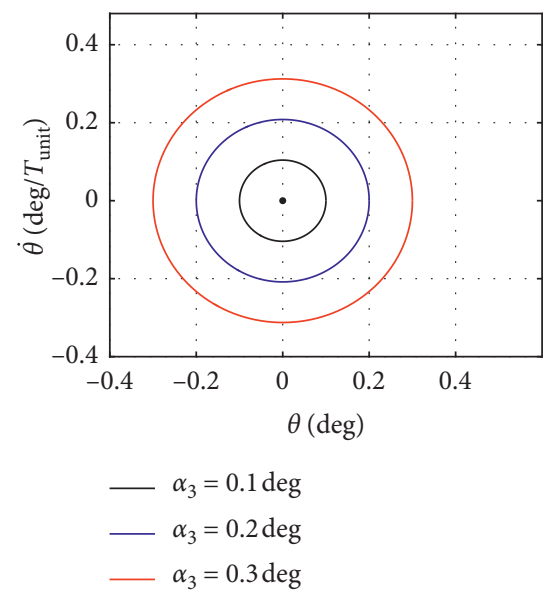

(c)

Figure 3: Linear periodic attitudes for a rigid spacecraft at libration point L2 in the Earth-Moon system. In all the following plots, the black dot stands for the equilibrium attitude.

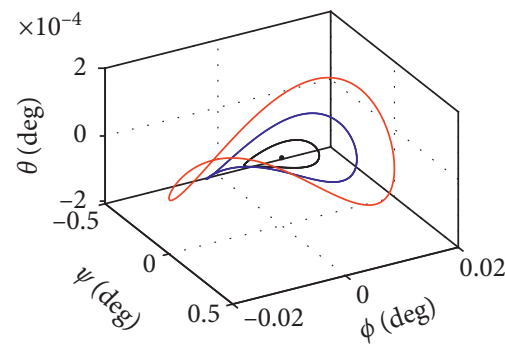

(a)

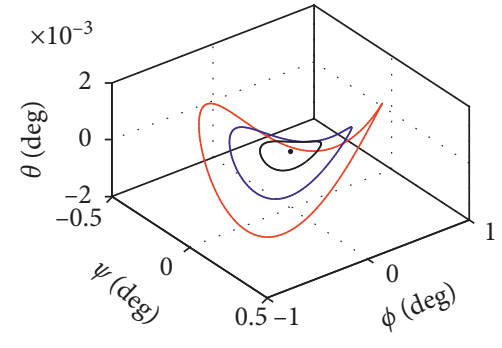

(b)

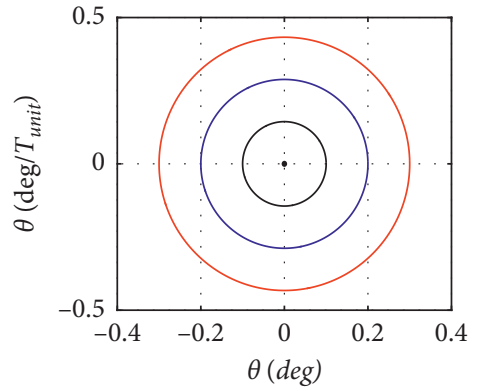

(c)

FIGURE 4: The periodic attitudes obtained by the differential correction method for a rigid spacecraft at libration point L2 in the Earth-Moon system. 


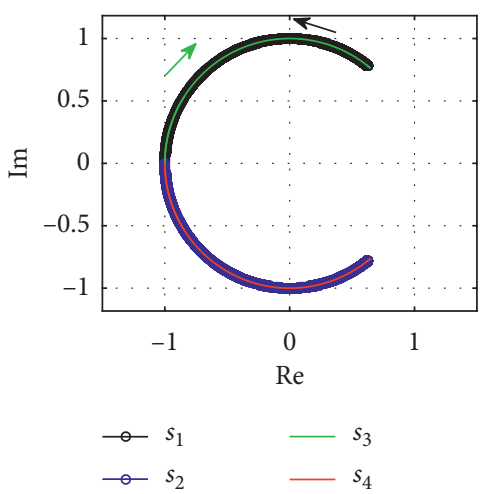

(a)

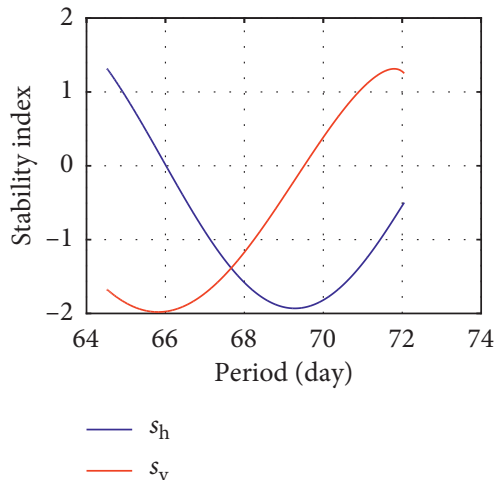

(b)

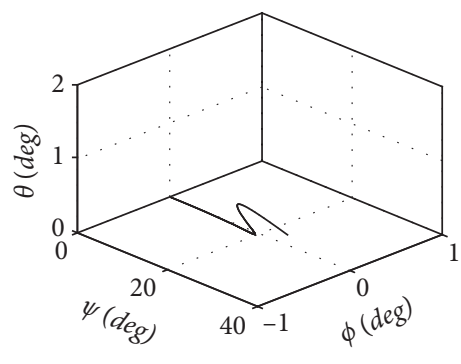

(c)

FIGURE 5: Stabilities of periodic attitudes in the first attitude family for a rigid spacecraft at libration point L2 in the Earth-Moon system. The eigenvalues of $s_{1}, s_{2}, s_{3}$, and $s_{4}$ of each periodic attitude are shown in the left panel, the stability index $s_{h}=s_{1}+s_{2}$ and $s_{v}=s_{3}+s_{4}$ are given in the middle one, and the Euler angles are shown in the right one.

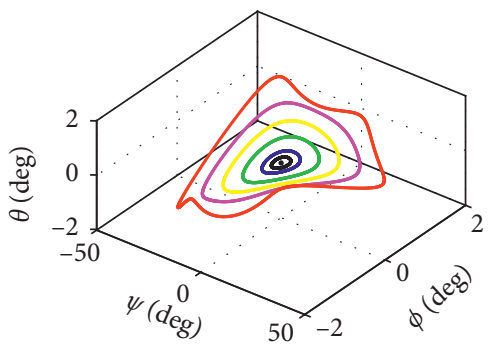

(a)

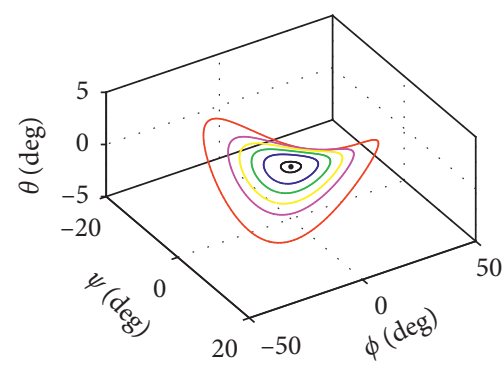

(b)

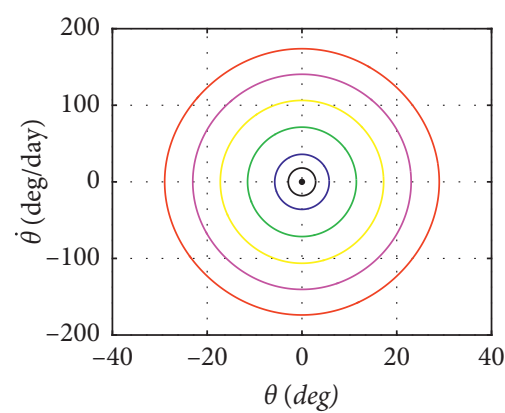

(c)

Figure 6: Three families of periodic attitudes for a rigid spacecraft at libration point L2 in the Earth-Moon system.

$$
\begin{aligned}
L & =\frac{5}{2}+\frac{3}{4} \sqrt{1+a^{2}}, \\
M & =\frac{5}{2}-\frac{3}{4} \sqrt{1+a^{2}},
\end{aligned}
$$

with

$$
a=-\tan 2 \theta_{E}
$$

By analysing the characteristic equations of (16), the stability conditions can be written as $[25,28]$

$$
\left\{\begin{array}{l}
C^{2}-D>0, \\
C>0, D>0, \\
k_{3}<0,
\end{array}\right.
$$

where $C=k_{1} k_{2}+(M-1) k_{2}+(L-1) k_{1}+1 \quad$ and $D=4 L M k_{1} k_{2}$.

Then, the stability diagram on the $k_{2}-k_{1}$ plane for spacecrafts located at L4 is shown in Figure 2(b), and coloured regions III and IV represent that the equilibrium attitude is stable with the corresponding mass distribution. The stable domains are distributed in the first and third quadrants, similar to the conclusions given by Guzzetti and
Howell, Robinson, and Wong et al. [25, 28, 31]. The stability domain in the first quadrant is a triangular region which represents $k_{1}>k_{2}>0\left(I_{z z}>I_{x x}>I_{y y}\right)$, and in the third quadrant, the stability domain represents $0>k_{1}>k_{2}\left(I_{x x}>I_{y y}>I_{z z}\right)$.

3.2.2. Periodic Attitudes and Their Stability. In this part, we take a spacecraft of the mass distribution in region III with $k_{1}=0.4$ and $k_{2}=0.2$ as an example. The linear solution of attitude motion around the equilibrium attitude $\left(\psi_{E}, \phi_{E}, \theta_{E}\right)$ can also be written in a similar form to (13), where $\alpha_{1}, \alpha_{2}$, and $\alpha_{3}$ are the amplitudes and $\varphi_{10}, \varphi_{20}$, and $\varphi_{30}$ are the initial phase angles. Different with the case of L2, the frequencies $\omega_{1}, \omega_{2}$, and $\omega_{3}$ here are determined by

$$
\left\{\begin{array}{l}
\omega_{1}=\frac{\sqrt{2}}{2} \sqrt{C-\sqrt{C^{2}-D}}, \\
\omega_{2}=\frac{\sqrt{2}}{2} \sqrt{C+\sqrt{C^{2}-D}}, \\
\omega_{3}=\sqrt{(2 M-5) k_{3}},
\end{array}\right.
$$


and the constant coefficients are determined by

$$
\begin{aligned}
& \bar{\kappa}_{1}=\frac{\omega_{1}^{2}-L k_{1}}{\left(k_{1}-1\right) \omega_{1}}, \\
& \bar{\kappa}_{2}=\frac{\omega_{2}^{2}-L k_{1}}{\left(k_{1}-1\right) \omega_{2}} .
\end{aligned}
$$

According to (13), there are also three types of basic periodic attitudes emanating from the equilibrium attitude. By the pseudo-arclength continuation method, the corresponding attitude families are obtained shown in the Euler angle $(\psi, \phi, \theta)$ space (see the second family in Figure $7(\mathrm{c})$ ). As it is shown in Figure 8, three families of periodic attitudes are constructed. The attitude periods of the families are extended in the range of [70.326, 92.214] days, [18.953, 26.250] days, and $[34.120,56.691]$ days, respectively.

We also investigated the stability of these periodic attitude motions. For the second family, two pairs of eigenvalues denoted by $\left(s_{1}, s_{2}\right)$ and $\left(s_{3}, s_{4}\right)$ are shown in Figure 7(a), and two stability indices $s_{h}=s_{1}+s_{2}$ and $s_{v}=$ $s_{3}+s_{4}$ are shown in Figure 7(b). As we can see from these figures, both stable and unstable attitude solutions exist in the second family. And, the bifurcation points can be obtained corresponding to $s_{h}=2$ or $s_{v}=2$ and $s_{h}=-2$ or $s_{v}=-2$, and the bifurcation families may be obtained from the bifurcation points (see the bifurcation point $A$ in Figure $7(\mathrm{c})$ for the second family corresponding to $s_{h}=2$ ). The Euler angles along the evolution of the second attitude family are shown in Figure 7(c), accordingly, which consists of the stable (black) and unstable (red) attitude solutions.

\section{Applications}

The attitude solutions of a spacecraft located at libration points L2 and L4 have been discussed in Section 3. They may be considered in many interesting mission applications. As mentioned by Guzzetti and Howell [25], the body axis of the vehicles at the libration points is designed to maintain approximately along with the line through the primaries for power generation or direct communication. Thus, the orientation of a spacecraft at a libration point should be discussed.

Also, the spacecraft is not probable placed at a libration point in a space mission but rather moves along a periodic orbit around the libration points [25]. Generally speaking, the spacecraft on the periodic orbit may have similar attitude characteristics to that at the libration point. Thus, we aim to construct periodic attitude motion of a spacecraft moving along the libration point orbit starting with the solutions at the corresponding libration point.

In this section, both of the above problems are studied by taking libration point L4 as an example.

4.1. Application to the Sun Orientation. The attitude motions of a spacecraft located at libration point L4 have been obtained in Section 3.2, and it can be discussed to space orientation.
Firstly, for the axisymmetric spacecraft $\left(I_{x x}=I_{y y}\right.$, i.e., disk-like vehicle) whose axis of symmetry orthogonal to the orbital plane, it can be spinning about this axis with any arbitrary spin velocity [25] within the CRTBP. Moreover, the spacecraft never changes its initial orientation for an observer fixed in the rotating frame if the spin velocity about the axis of symmetry equals the angular rate of the rotating frame [33]. In the Earth-Moon CRTBP, the orientation can maintain alignment with the Sun if we adjust the spin velocity of the spacecraft equals to the Sun's angle rate. Thus, the spacecraft can be used in the Sun orientation, in this special case.

Then, following the attitude solutions corresponding to the third family of the spacecraft with $I_{x x} \neq I_{y y}$ in Section 3.2, we study how the solutions can be employed to the Sun orientation. The rotating period of the Sun around the EarthMoon centre is approximately 29.49 days, and the period of attitude motion of the spacecraft is in the range of [34.120, 56.691] days. Obviously, the principle axes of the spacecraft cannot always align with the Sun, but the periodic alignment may exist. Subsequently, choosing the period of its attitude motion with 39.32 days, the direction of the principle $x_{B}$-axis of the spacecraft, the position angle of the Sun aligning at the initial time, and their relationship in evolution are shown in Figure 9; cross points of the blue curves and the black lines mean the alignment of the spacecraft with the Sun. A periodic relationship of the spacecraft and the Sun occurs with a resonant ratio $N_{1}: N_{2}=4: 3$ (refer to Figure 9) implying that the Sun accomplishes $N_{1}$ revolutions about the EarthMoon barycentre, and the spacecraft requires to complete $N_{2}$ periods of the attitude motion. Other periodic relationships of $N_{1}: N_{2}$ can be obtained similarly. The blue curve represents the direction of the principle $x_{B}$-axis of the spacecraft at libration point L4; the black lines represent the position angle of the Sun in the Earth-Moon coordinate system, and the red dashed lines denote the boundary of their each resonant period. With this periodic relationship, the Sun orientation will be realized.

\subsection{Application to the Earth-Moon Libration Point Orbits}

4.2.1. Method. The amplitude $A_{m}$ adim of a target periodic orbit is defined along the direction from reference origin point $o$ to libration point L4. Then, a parameter $\sigma$ within the range of $[0,1]$ is introduced to characterize the amplitude of its corresponding orbit.

Observing the amplitude $\sigma A_{m}$, one can get that the case of $\sigma=0$ represents the libration point at that the attitude of a rigid body has been studied in Section 3, the case of $\sigma=1$ corresponds to the target libration point orbit, and the case of $\sigma \in(0,1)$ corresponds to the intermediate orbit. By continuing from $\sigma=0$ to $\sigma=1$, the orbit can transit from a libration point to the target periodic orbit. Meanwhile, the attitude motion can be adjusted gradually starting from a libration point to the aiming periodic orbit. Based on this continuation method, the attitude motion of a rigid spacecraft moving along a libration point orbit with amplitude of $A_{m}$ can be constructed. The solutions are helpful 


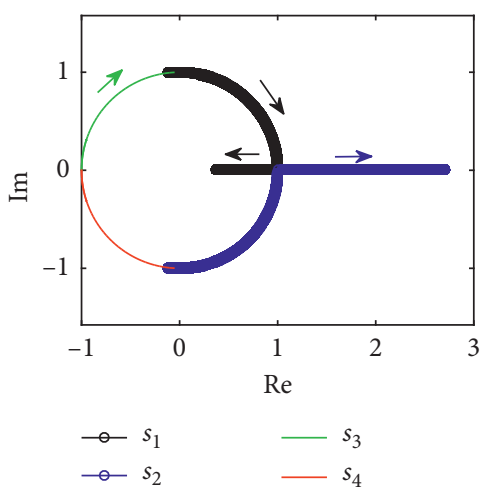

(a)

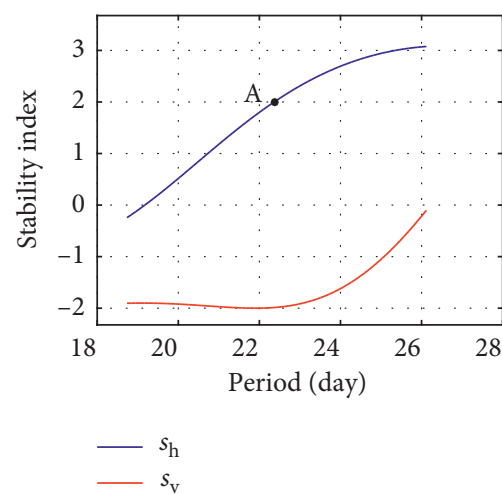

(b)

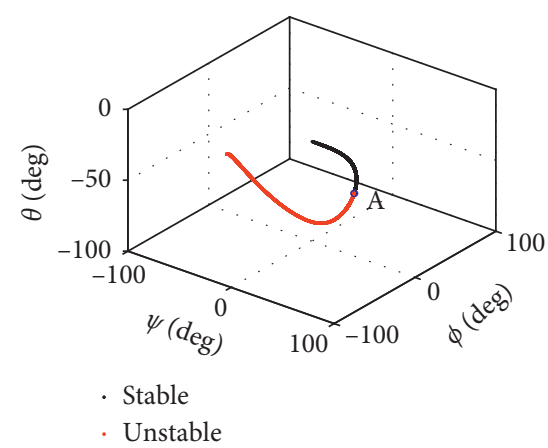

(c)

FIGURE 7: Stabilities of periodic attitudes in the second attitude family for a rigid spacecraft at libration point L4 in the Earth-Moon system.

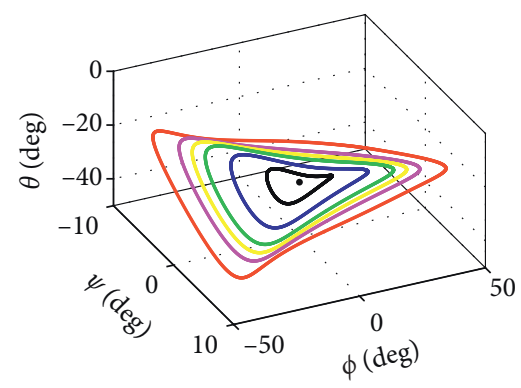

(a)

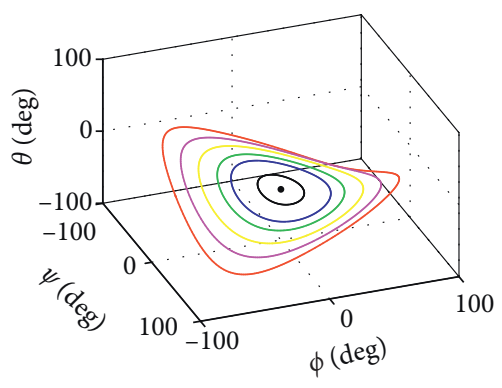

(b)

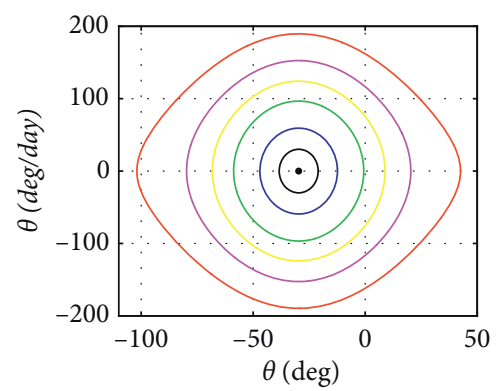

(c)

Figure 8: Three families of periodic attitudes for a rigid spacecraft at libration point L4 in the Earth-Moon system.

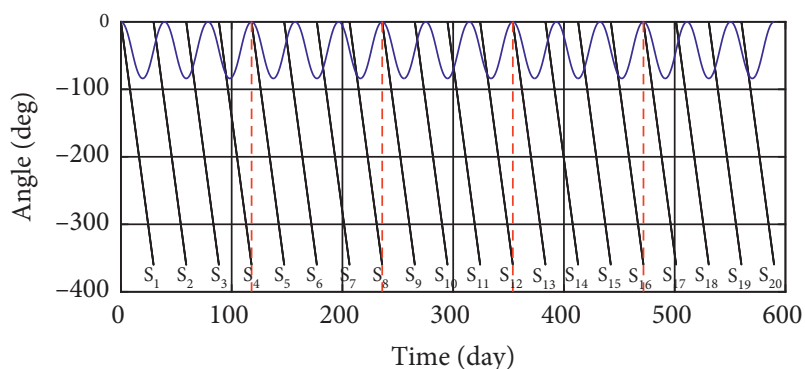

FIgURE 9: The direction of a spacecraft (blue curve) at libration point L4 and the position angle of the Sun (black line) measured in the Earth-Moon coordinate system. The cross points of the blue curve and the black lines mean the alignment of the spacecraft with the Sun, and the red dashed lines denote the resonant period.

in understanding the natural rotational motion which may offer options that reduce the control effort for the attitude control system in a number of applications.

4.2.2. Orbit-Attitude Motion. Orbit-attitude behaviours that are periodic in the Earth-Moon CRTBP rotating frame are the main topic of this part. In this section, the planar libration point orbit of L 4 with amplitude 0.01 in normalized unit is adopted as an example to construct the attitude motions of a spacecraft along it. Using the continuation process, attitude motions of a spacecraft from libration point L4 to the corresponding periodic orbit can be constructed. In the process of continuation, differential corrector is used, and the procedure to construct attitude motions along the other libration point orbits is similar.

The period of libration point orbit of L4 with amplitude 0.01 is approximately 28.58 days. Then, compare it with the periods of the attitude motions at libration point L 4 shown in Section 3.2. As we can see, the periods of the attitude motion are extended from 18.953 days to 92.214 days. There exists one type of periodic motion, orbit-attitude resonant motion. In our study, several orbit-attitude resonances are found which satisfied $N_{1} \cdot T_{\text {attitude }}=N_{2} \cdot T_{\text {orbit }}$. The equation implies that the spacecraft accomplishes $N_{1}$ attitude motion revolutions in the same time interval that $N_{2}$ orbital motions along the libration point orbit are required. The process is as follows: (1) comparing the periods of the attitude motion and the orbit of the spacecraft, the suitable terms of $N_{1}$ and $N_{2}$ can be obtained straightforwardly. (2) Through the method introduced in Section 4.2.1, the corresponding orbit-attitude resonant motion would be constructed gradually. (3) The results of orbit-attitude resonances with $N_{1} \cdot T_{\text {attitude }}=N_{2} \cdot T_{\text {orbit }}$ will be validated and shown. In this part, for the amplitude 0.01 , the resonance of $N_{1}: N_{2}=1: 1$ corresponding to the planar case and $N_{1}: N_{2}=1: 3$ and $2: 5$ corresponding to the threedimensional case is shown in Figure 10. Other kinds of 


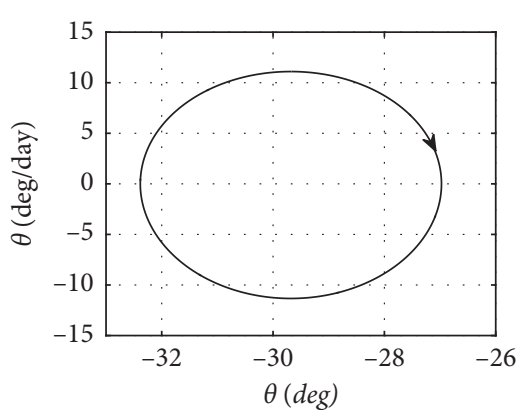

(a)

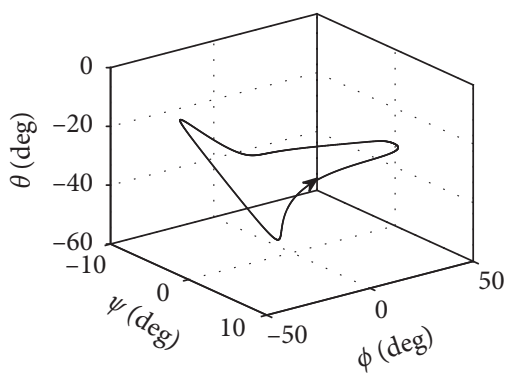

(b)

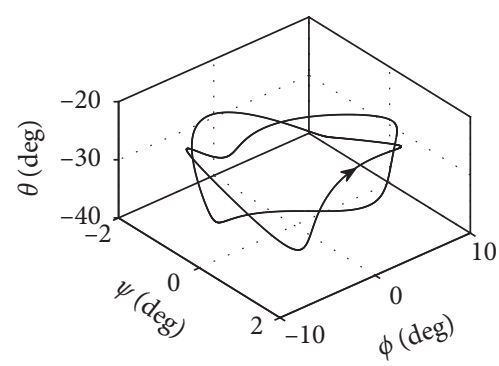

(c)

FIGURE 10: Orbit-attitude resonances for a rigid spacecraft along a periodic orbit around libration point L4 in the Earth-Moon system.

resonance between orbit and attitude motion may exist, but they are not shown in this paper.

\section{Conclusions}

The overall goal of this paper is providing a better understanding about the attitude motion of a rigid spacecraft within the circular restricted three-body problem. In the Earth-Moon CRTBP, the equilibrium attitude of a spacecraft and its stability are obtained when the spacecraft is fixed at the libration points L2 or L4. For a spacecraft in the stability domain, three families of periodic attitude motions around the equilibrium attitude are constructed by the pseudo arclength continuation method. These solutions are applicable to the Sun orientation, and with these solutions as initial guesses, several orbit-attitude resonances are constructed when spacecrafts are located on periodic orbits. The method to obtain periodic attitude motion along a libration point orbit in this paper may be employed to identify potential natural behaviours in other dynamical models.

\section{Data Availability}

The data used to support the findings of this study are available from the corresponding author upon request.

\section{Conflicts of Interest}

The authors declare that they have no conflicts of interest.

\section{Acknowledgments}

This work was performed with the financial support of the Basic Scientific Research Fund of National Defense (no. 2016110C019), the National Basic Research Program 973 of China (no. 2015CB857100), the Natural Science Foundation of Jiangsu Province (nos. BK20160612 and BK20160811), and the National Natural Science Foundation of China (nos. 11603011 and 61803027).

\section{References}

[1] G. Gómez, M. W. Lo, and J. J. Masdemont, Libration Point Orbits and Applications, WSP, Singapore, 2003.

[2] I. A. Robin and V. V. Markellos, "Numerical determination of three-dimensional periodic orbits generated from vertical self-resonant satellite orbits," Celestial Mechanics, vol. 21, no. 4, pp. 395-434, 1980.

[3] C. G. Zagouras and P. G. Kazantzis, "Three-dimensional periodic oscillations generating from plane periodic ones around the collinear Lagrangian points," Astrophysics and Space Science, vol. 61, no. 2, pp. 389-409, 1979.

[4] K. Howell, "Families of orbits in the vicinity of the collinear libration points," in Proceedings of the AIAA/AAS Astrodynamics Specialist Conference and Exhibit, Boston, MA, USA, August 1998.

[5] R. W. Farquhar and A. A. Kamel, "Quasi-periodic orbits about the translunar libration point," Celestial Mechanics, vol. 7, no. 4, pp. 458-473, 1973.

[6] J. V. Breakwell and J. V. Brown, "The "Halo" family of 3dimensional periodic orbits in the Earth-Moon restricted 3body problem," Celestial Mechanics, vol. 20, no. 4, pp. 389404, 1979.

[7] M. Popescu and V. Cardoş, "The domain of initial conditions for the class of three-dimensional halo periodical orbits," Acta Astronautica, vol. 36, no. 4, pp. 193-196, 1995.

[8] G. Gómez, Dynamics and Mission Design Near Libration Points: Fundamentals-The Case of Collinear Libration Points, World Scientific, Singapore, 2001.

[9] X. Y. Hou and L. Liu, "On motions around the collinear libration points in the elliptic restricted three-body problem," Monthly Notices of the Royal Astronomical Society, vol. 415, no. 4, pp. 3552-3560, 2011.

[10] H. Lei and B. Xu, "High-order analytical solutions around triangular libration points in the circular restricted three-body problem," Monthly Notices of the Royal Astronomical Society, vol. 434, no. 2, pp. 1376-1386, 2013.

[11] H. Lei, B. Xu, X. Hou, and Y. Sun, "High-order solutions of invariant manifolds associated with libration point orbits in the elliptic restricted three-body system," Celestial Mechanics and Dynamical Astronomy, vol. 117, no. 4, pp. 349-384, 2013.

[12] M. Xu, Y. Liang, and K. Ren, "Survey on advances in orbital dynamics and control for libration point orbits," Progress in Aerospace Sciences, vol. 82, pp. 24-35, 2016.

[13] Y. J. Qian, X. D. Yang, L. Y. Yang et al., "Approximate analytical methodology for the restricted three-body and fourbody models based on polynomial series," International Journal of Aerospace Engineering, vol. 2016, Article ID 9747289, 8 pages, 2016.

[14] Y. J. Qian, X. D. Yang, G. Q. Zhai et al., "Analytical and numerical construction of vertical periodic orbits about triangular libration points based on polynomial expansion relations among directions," Astrophysics and Space Science, vol. 362, no. 8, p. 136, 2017. 
[15] Y.-J. Qian, X.-D. Yang, W. Zhang, and G.-Q. Zhai, "Periodic motion analysis around the libration points by polynomial expansion method in planar circular restricted three-body problem," Nonlinear Dynamics, vol. 91, no. 1, pp. 39-54, 2018.

[16] Y. Qian, X. Yang, W. Jing, and W. Zhang, "An improved numerical method for constructing Halo/Lissajous orbits in a full solar system model," Chinese Journal of Aeronautics, vol. 31, no. 6, pp. 1362-1374, 2018.

[17] Y.-J. Qian, X.-D. Yang, G.-Q. Zhai, and W. Zhang, "Planar periodic orbits' construction around libration points with invariant manifold technique," Proceedings of the Institution of Mechanical Engineers, Part G: Journal of Aerospace Engineering, vol. 233, no. 2, pp. 498-509, 2019.

[18] R. Farquhar, "The flight of ISEE-3/ICE-Origins, mission history, and a legacy," in Proceedings of the AIAA/AAS Astrodynamics Specialist Conference and Exhibit, Boston, MA, USA, August 1998.

[19] V. Domingo, B. Fleck, and A. I. Poland, "The SOHO mission: an overview," The SOHO Mission, vol. 162, no. 1-2, pp. 1-37, 1995.

[20] F. L. Markley, S. F. Andrews, J. R. O’Donnell, and D. K. Ward, "Attitude control system of the wilkinson microwave anisotropy probe," Journal of Guidance, Control, and Dynamics, vol. 28, no. 3, pp. 385-397, 2005.

[21] J. Minow, R. Altstatt, and W. Skipworth, "Genesis radiation environment," in Proceedings of the 45th AIAA Aerospace Sciences Meeting and Exhibit, Reno, NV, USA, January 2007.

[22] M. Giard and L. Montier, "Investigating clusters of galaxies with planck and herschel," $3 K, S N$ 's, Clusters: Hunting the Cosmological Parameters with Precision Cosmology, vol. 290, no. 1-2, pp. 159-166, 2004.

[23] C. Cacciari, Mapping the Stellar Populations of the Milky Way with Gaia, Asteroseismology of Stellar Populations in the Milky Way, Springer, Cham, Switzerland, 2015.

[24] S. Hu, C. Sun, and Y. Liu, "Orbit design for satellite Kuafu-a based on invariant manifolds," Aerospace Control, vol. 27, no. 3, pp. 37-41, 2009.

[25] D. Guzzetti and K. C. Howell, "Attitude dynamics in the circular restricted three-body problem," Astrodynamics, vol. 2, no. 2, pp. 87-119, 2018.

[26] T. R. Kane and E. L. Marsh, "Attitude stability of a symmetric satellite at the equilibrium points in the restricted three-body problem," Celestial Mechanics, vol. 4, no. 1, pp. 78-90, 1971.

[27] W. J. Robinson, "The restricted problem of three bodies with rigid dumb-bell satellite," Celestial Mechanics, vol. 8, no. 2, pp. 323-330, 1973.

[28] W. J. Robinson, "Attitude stability of a rigid body placed at an equilibrium point in the restricted problem of three bodies," Celestial Mechanics, vol. 10, no. 1, pp. 17-33, 1974.

[29] A. Abad, M. Arribas, and A. Elipe, "On the attitude of a spacecraft near a Lagrangian point," Bulletin of the Astronomical Institutes of Czechoslovakia, vol. 40, no. 5, pp. 302-307, 1989.

[30] E. Brucker and P. Gurfil, "Analysis of gravity-gradient-perturbed rotational dynamics at the collinear Lagrange points," The Journal of the Astronautical Sciences, vol. 55, no. 3, pp. 271-291, 2007.

[31] B. Wong, R. Patil, and A. Misra, "Attitude dynamics of rigid bodies in the vicinity of the Lagrangian points," Journal of Guidance, Control, and Dynamics, vol. 31, no. 1, pp. 252-256, 2008.

[32] A. J. Knutson, D. Guzzetti, K. C. Howell, and M. Lavagna, "Attitude responses in coupled orbit-attitude dynamical model in earth-moon Lyapunov orbits," Journal of Guidance, Control, and Dynamics, vol. 38, no. 7, pp. 1264-1273, 2015.
[33] D. Guzzetti and K. C. Howell, "Natural periodic orbit-attitude behaviors for rigid bodies in three-body periodic orbits," Acta Astronautica, vol. 130, pp. 97-113, 2017.

[34] Y. Meng, R. Hao, and Q. Chen, "Attitude stability analysis of a dual-spin spacecraft in halo orbits," Acta Astronautica, vol. 99, pp. 318-329, 2014.

[35] L. Bucci, M. Lavagna, D. Guzzetti, and K. C. Howell, "Periodic orbit-attitude solutions along planar orbits in a perturbed circular restricted three-body problem for the Earth-Moon system," Acta Astronautica, vol. 147, pp. 152-162, 2018.

[36] A. Colagrossi and M. Lavagna, "Preliminary results on the dynamics of large and flexible space structures in Halo orbits," Acta Astronautica, vol. 134, pp. 355-367, 2017.

[37] H. Lei, C. Circi, E. Ortore et al., "Periodic attitudes and bifurcations of a rigid spacecraft in the second degree and order gravity field of a uniformly rotating asteroid," Celestial Mechanics and Dynamical Astronomy, vol. 130, no. 8, p. 53, 2018.

[38] S. Gong, J. Li, and J. Simo, "Orbital motions of a solar sail around the L2 earth-moon libration point," Journal of Guidance, Control, and Dynamics, vol. 37, no. 4, pp. 13491356, 2014.

[39] D. Tamakoshi and H. Kojima, "Solar sail orbital control using reflectivity variations near the earth-moon L2 point," Journal of Guidance, Control, and Dynamics, vol. 41, no. 2, pp. 417430, 2018 .

[40] J. Heiligers, S. Hiddink, R. Noomen, and C. R. McInnes, "Solar sail Lyapunov and halo orbits in the earth-moon three-body problem," Acta Astronautica, vol. 116, pp. 25-35, 2015.

[41] Y.-J. Qian, Z.-X. Liu, X.-D. Yang, I. Hwang, and W. Zhang, "Novel subharmonic resonance periodic orbits of a solar sail in earth-moon system," Journal of Guidance, Control, and Dynamics, vol. 42, no. 11, pp. 2532-2540, 2019.

[42] V. Szebehely, Theory of Orbits: The Restricted Problem of Three Bodies, Academic Press, NewYork, NY, USA, 1967.

[43] E. J. Doedel, R. C. Paffenroth, H. B. Keller, D. J. Dichmann, J. Galán-Vioque, and A. Vanderbauwhede, "Computation of periodic solutions of conservative systems with application to the 3-body problem," International Journal of Bifurcation and Chaos, vol. 13, no. 6, pp. 1353-1381, 2003.

[44] H. Lei, C. Circi, E. Ortore, and B. Xu, "Attitude stability and periodic attitudes of rigid spacecrafts on the stationary orbits around asteroid 216 Kleopatra," Advances in Space Research, vol. 63, no. 2, pp. 1017-1037, 2019.

[45] W. E. Wiesel and D. J. Pohlen, "Canonical floquet theory," Celestial Mechanics \& Dynamical Astronomy, vol. 58, no. 1, pp. 81-96, 1994.

[46] M. Hénon, "Numerical exploration of the restricted problem, V," Astronomy and Astrophysics, vol. 1, pp. 223-238, 1969.

[47] M. Hénon, "Vertical stability of periodic orbits in the restricted problem," Celestial Mechanics and Dynamical Astronomy, vol. 8, no. 2, pp. 269-272, 1973.

[48] M. Hénon, "Vertical stability of periodic orbits in the restricted problem. I. Equal masses," Astronomy and Astrophysics, vol. 28, p. 415, 1973. 